MODELING, IDENTIFICATION AND CONTROL, 1996, VOL. 17, NO. 1, 37-45

doi:10.4173/mic.1996.14

\title{
Model based teleoperation of untethered underwater vehicles with manipulators, MOBATEL
}

\author{
JENS G. BALCHEN† \\ Keywords: Model based, teleoperation, underwater vehicle
}

Model based teleoperation of underwater vehicles and manipulators is a way to solve the problem introduced by the very long delay caused by the narrow band sonic communication link between the vehicle and the surface vessel. The MOBATEL project is described.

\section{Introduction}

The MOBATEL-project is a long range research programme at the Norwegian Institute of Technology sponsored by the The Research Council of Norway in cooperation with a number of Norwegian industrial organizations.

Eleven doctoral candidates at the Norwegian Institute of Technology, Department of Engineering Cybernetics and Department of Production and Quality Engineering do their research projects on different subsystems of the total MOBATEL system. These will be referred to under the detailed discussions below (Balchen and Yin 1994).

The programme aims at developing the basic concepts and some of the key technologies for remote control of a free-swimming underwater vehicle from a surface vessel towards a deep-sea landscape of objects as illustrated in Fig. 1.

A free-swimming, untethered underwater vehicle which can operate at great depths is useful for a number of purposes. Such purposes can be inspection, data collection (as a carrier of a complex detection system), handling of objects, assembly, teleoperation of equipment, simple machining operations, etc. A number of these operations assume that the vehicle is equipped with one or more manipulator arms (robot arms).

The main problem dealt with in the MOBATEL-project is that there is no cable between the vehicle and the surface vessel for communication and that the only communication thus will be via a sonic (ultra-sonic) link. This has great operational advantages with respect to manoeuvrability in bad weather, under and inside complicated structures, etc. On the other hand, the very narrow band communication link (around $100 \mathrm{bits} / \mathrm{s}$ ) constitutes a serious problem for telecontrol (teleoperation). This is the main motivation for the development of a model based control system.

The problems encountered in remote control over a narrow band communication channel (or broad band communication channel with large delays like in space technology) are general and universal. These problems have been studied theoretically for a long time and good solutions are known. What is new today is the availability of

Received 20 August 1995

Department of Engineering Cybernetics, the Norwegian Institute of Technology, 7034 Trondheim, Norway.

Presented at the 3rd IFAC Workshop on Control Applications in Marine Systems, May 1995, Trondheim. Norway (CAMS '95). 


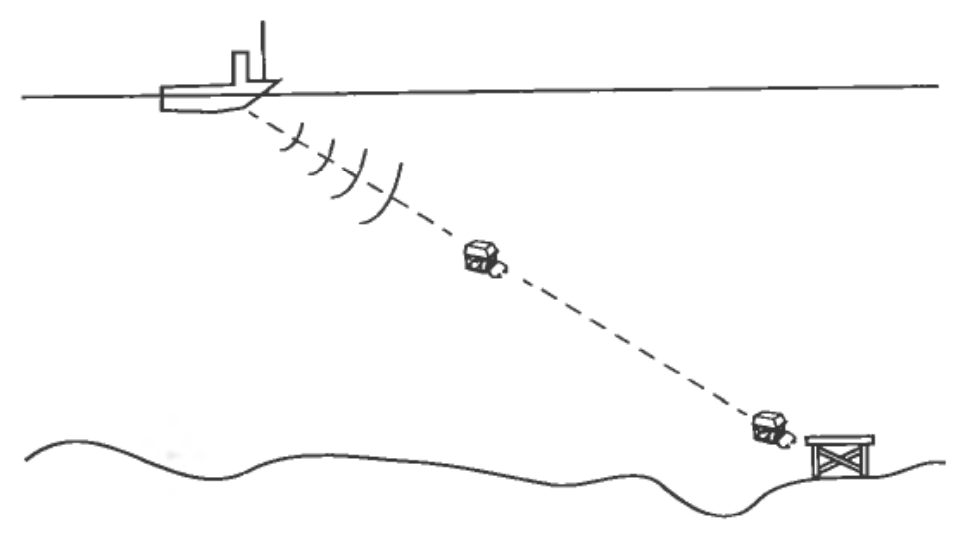

Figure 1.

high speed, high capacity, computing power at reasonable price. Therefore, the theoretical solutions can now find their practical realization.

\section{Why teleoperation or operator based remote control?}

Teleoperation means that there is a human operator with his skills, judgement, experience and ability to make decisions who performs the control operations. An alternative to teleoperation is autonomous control in which the vehicle has enough local 'intelligence' to perform control functions and make decisions without human intervention. Autonomous underwater vehicles (AUVs) are useful in many cases, e.g. long distance surveillance purposes. However, such aspects are not included in the MOBATEL-project. The use of human operators to control underwater operations will in most cases be quite natural and appropriate because the total replacement of the skills and judgement of operators by 'artificial intelligence' is not feasible today.

\section{Basic principles of model based teleoperation}

The MOBATEL project develops the system shown in fig. 2 . On the left side two operators are seen in the cockpit of the surface vessel. The 'image operator' has the function of updating the computer-generated and model-based images of the landscape objects, so that they fit with the compressed camera images received from the vehicle. 'The pilot' steers the 'vehicle model' in the landscape displayed on his monitor based on the images created by the image operator.

The images displayed on the screens are generated synthetically be a graphics computer (Silicon Graphics IRIS 4D/320VGX) and they constitute what the operators would have seen had they been sitting on board the vehicle and looking through a window. These images are not delayed by the low bandwidth ultrasonic transmission because they are generated by mathematical models programmed in the computer which is near the operator and is part of the system. The control signals generated by the pilot which are related to the six DOF motion of the vehicle and possibly also the motion of one or more manipulator arms, are lead to the ultrasonic communication system for transmission to the vehicle and also to the simulation model computing the motion of 


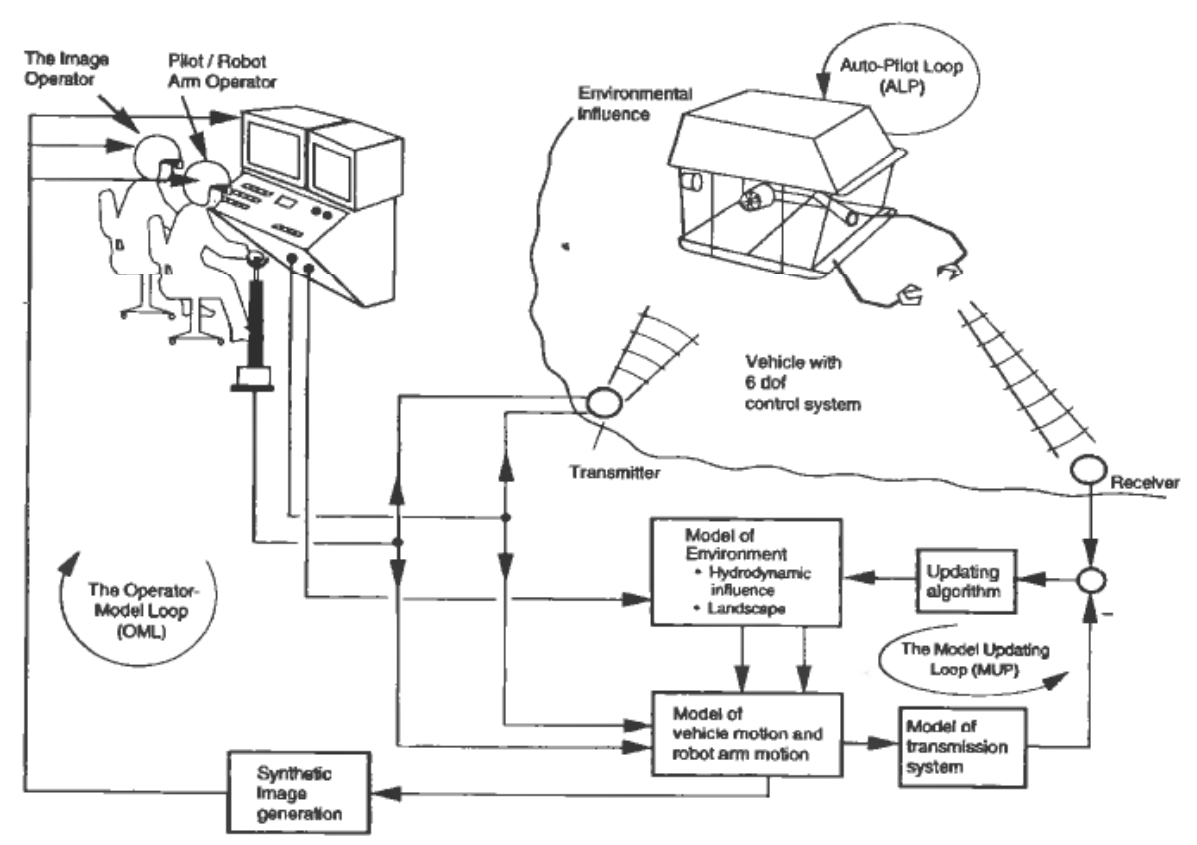

Figure 2.

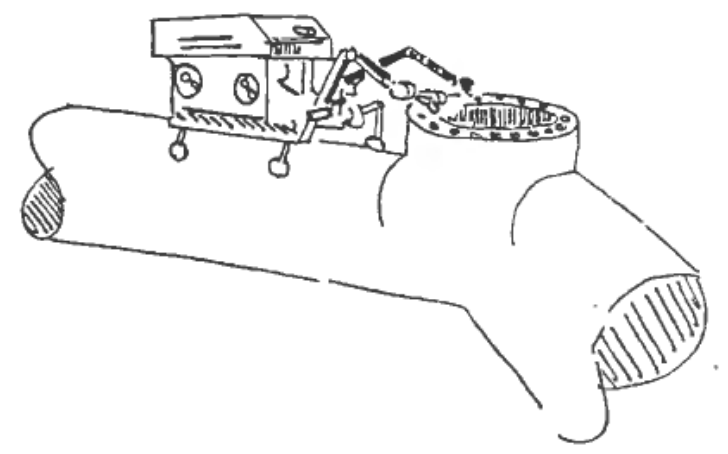

Figure 3.

the vehicle. The delay and the bandwidth limitation associated with the transmission down to the vehicle must be considered together with the equivalent transmission of signals back from the vehicle. The combined effects of these transmission properties are represented by one block in the model after the dynamics of the vehicle.

The MOBATEL project tries to utilize standard industrial products available on the market for most functions. An example of this is the ultrasonic communication system which has been developed by one of the industrial participants. It has a communication capacity of $100 \mathrm{bits} / \mathrm{s}$ for the time being.

The system can be regarded as having two modes of operation

- The free swimming mode which has the purpose of controlling the vehicle motion to within an accuracy of say $0.1-0.5 \mathrm{~m}$ for the purpose of inspection etc. and docking the vehicle on a solid surface. 
- After docking on a solid surface (Fig. 3) in the manipulator control mode to perform useful tasks of assembly, machining etc.

\section{The freeswimming mode}

In the free-swimming mode the vehicle has a local autopilot which is a 6 DOF model based control system (Fjellstad 1994). The hydrodynamic vehicle model is developed by Fossen (1991). The velocity setpoint for the 6 DOF autopilot are generated from the 4 DOF pilot control stick by a special algorithm (on the surface).

In some cases it is advantageous to the pilot to address only certain points on the desired vehicle trajectory and leave to the autopilot to generate an optimal trajectory between the points (Spangelo 1994).

The actual motion of the vehicle relative to the subsea landscape is observed by a number of devices primarily one or more underwater cameras mounted on the vehicle. The images generated by a camera have to be highly compressed before they are transmitted over the ultrasonic link. For this purpose a new compression algorithm named MOPIC, has been developed whose properties are optimized for the very narrow bandwidth of the link (100 bits/s) (Yin 1993).

An example of the quality of a received image by MOPIC compared with the standard JPEG algorithm is shown in Fig. 4.

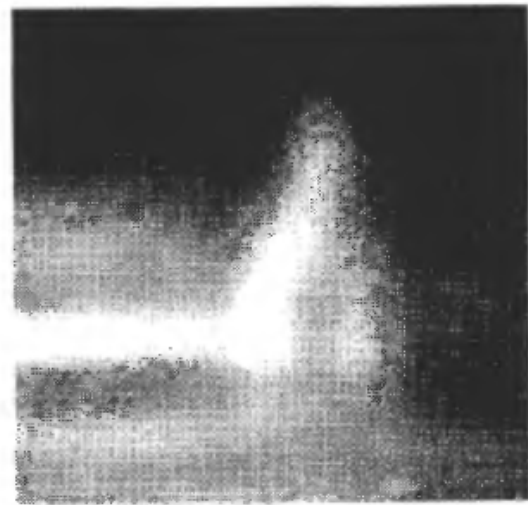

100 bytes

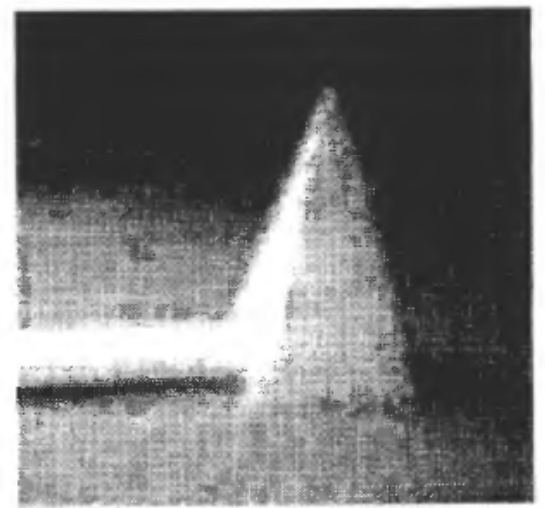

400 bytes

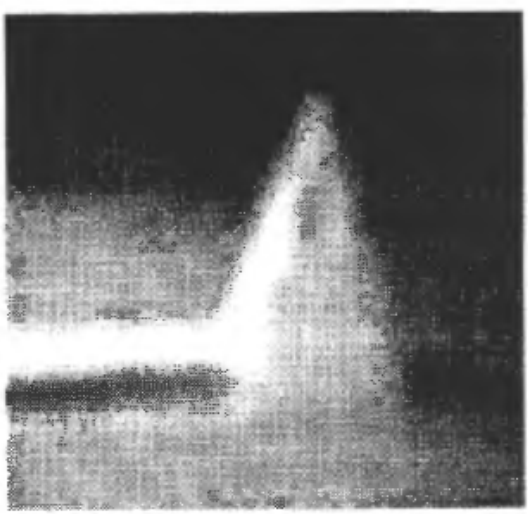

200 bytes

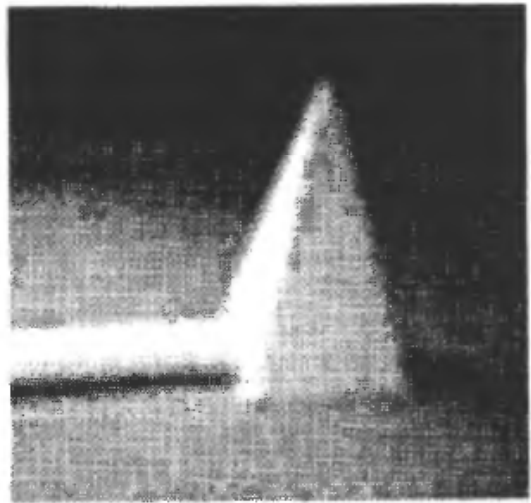

800 bytes

(a) 


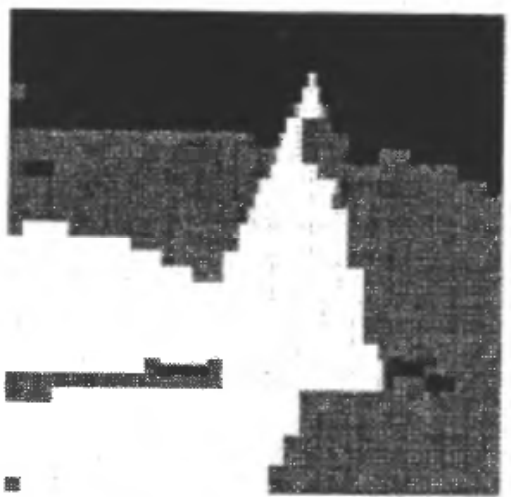

513 bytes

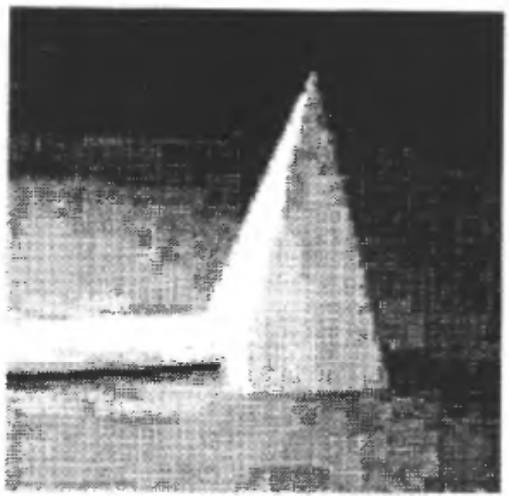

807 bytes

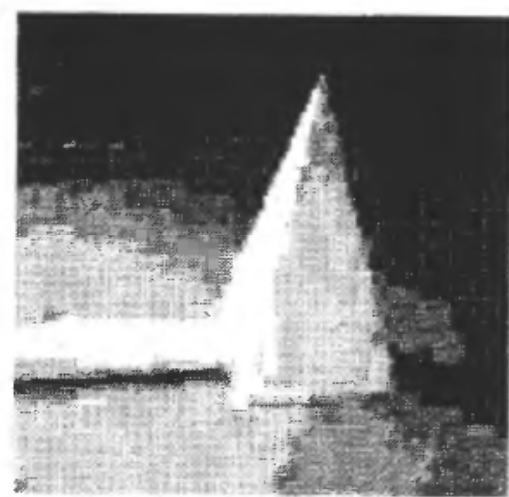

661 bytes

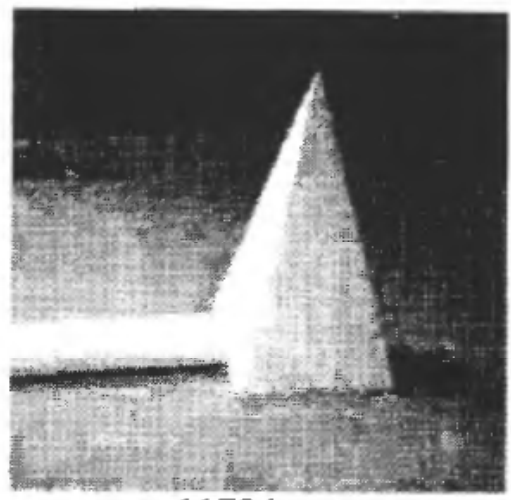

1173 bytes

(b)

Figure 4. (a) Images received by MOPIC. (b) Images received by JPEG.

The image operator has the function of interpreting the images received by MOPIC and fit them to images generated by the computer based on geometric object models from a data base. If the landscape is completely known and a CAD model exists the primary function of the image operator is to recognize where in the model the vehicle is located. If the landscape is fully or partly unknown the image operator must construct a landscape model based on elementary object models that are fit to the images. The latter function takes longer time.

The operator assisted image reconstruction (Volden 1995) is a part of the Model Updating Loop (MUL) shown in Fig. 1 and which contains the delay of the model of the transmission system.

One major cause of difference in behaviour of the real vehicle and the modeled vehicle is that the real vehicle is subject to an environmental influence caused by the motion of the surrounding water. Under certain conditions (depth greater than $100 \mathrm{~m}$, near horizontal bottom, far from objects etc.) the drift velocity may regarded as a constant 2 DOF vector which can be updated by the MUL. In more complicated environments a rotational influence must be considered in addition in the MUL (Ren 1993).

When the MUL works properly, the environmental model consisting of the hydrodynamic influence and the landscape objects together with the model of the 
vehicle motion will form the basis for generating the synthetic images of the landscape as viewed from the vehicle and to be displayed for the pilot. Thereby the Operator Model Loop (OML) is established which does not contain the delay of the ultrasound transmission system. This means that the pilot is able to control the synthetic vehicle with high bandwidth as if he were sitting in the real vehicle and controlling it directly.

The success of this control scheme is dependent upon many factors such as

- the quality of the model of controlled vehicle motion.

- the quality of the model of environment hydrodynamic influence.

In total these two items express the ability to predict the vehicle's motion to compensate for the delay in the Model Updating Loop (MUL).

- the quality of the operator assisted reconstruction of landscape objects and their synthetic real time stereo representation on the display of the pilot.

Assuming as an example that the model updating loop (MUL) has a repetition period of $30 \mathrm{~s}$ and that the maximal position error to be tolerated is $0.3 \mathrm{~m}$, the consequence is that the maximal total velocity error that can be tolerated is $0.01 \mathrm{~m} / \mathrm{s}=1 \mathrm{~cm} / \mathrm{s}$.

This order of magnitude error is believed to be achievable in a well tuned system with off the shelf inertial instrumentation in the autopilot, but it still has to be proven by actual field experiments.

One important factor influencing the total accuracy of navigation is the precision of the camera onboard the vehicle which generates the images to be transmitted to the surface vessel. Because the parameters of the camera geometric model may vary due to mechanical and thermal conditions, a technique for precise calibration of the camera is needed (Melen 1994).

In the initial approach mode the freeswimming vehicle is guided from the surface vessel to the subsea landscape (e.g. oil production equipment) by means of a sonar on the surface vessel as indicated in Fig. 1. The accuracy of this remote control can be secured by means of a transponder onboard the free swimming vehicle yielding a precise sonar echo. Thereby it is expected that the positional accuracy achievable may be around $\pm 5 \mathrm{~m}$ on a distance of $2000 \mathrm{~m}$. Thereby a transition from surface based remote control to local image based control is feasible. A local image based autopilot loop will perform as follows:

- An image of an object is captured by the camera.

- A very short message about this fact is transmitted to the operator on the surface who returns a command which makes the autopilot go into 'freeze mode'.

- In the 'freeze mode' the $6 \mathrm{DOF}$ autopilot setpoints are controlled so that the image generated by the camera does not change position, orientation or size.

- The new control loop operates in 'real time' with a sampling frequency around $24 \mathrm{~Hz}$.

- The main purpose of this 'freeze mode' is to give the image operator on the surface time to study and recognize the image of the object and to prepare his operations without the vehicle drifting away relative to the object.

The research on the 'freeze mode' system is not yet completed (Melen 1994).

\section{The manipulator control mode}

One or more manipulators mounted on the free swimming vehicle may be operated in two ways. 


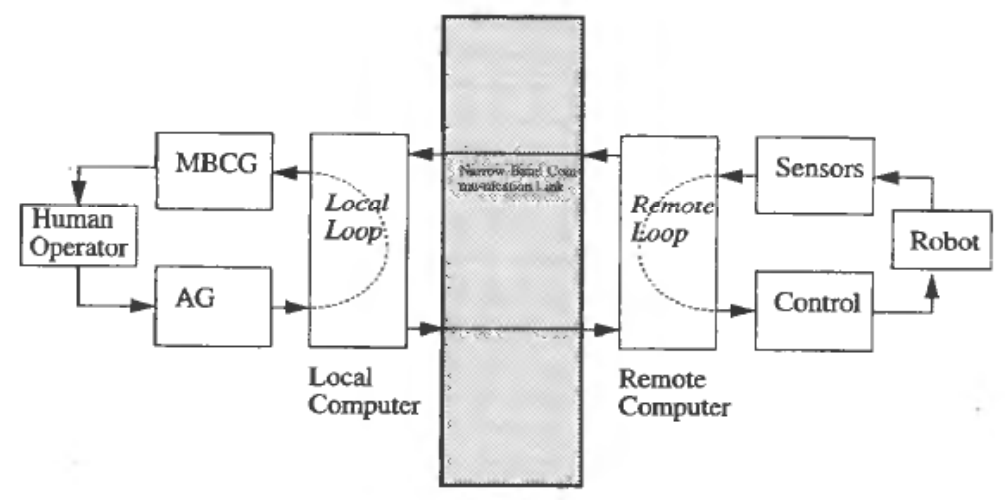

Figure 5.

- While the vehicle is free swimming, and

- While the vehicle is secured to some solid surface.

Obviously the achievable precision in the first of these modes is much lower than in the second mode for a number of reasons. One of these reasons is the interaction between the manipulator motion and the motion of the vehicle. This has been studied in detail by Schjølberg and Fossen (1994). Further research in this field is going on.

When the vehicle is secured to a rigid surface, a modelbased manipulator control system can be implemented (Tan 1993). The pilot on the surface is then replaced by an operator who has a 6 DOF anthropomorphic generator (joystick) at his disposal. As shown in fig. 5, the human operator observes a stereo image of the manipulator arm and hand on the MBCG (Model Based Computer Graphics) and controls a dynamic model of the manipulator by means of the AG (anthropomorphic generator) to perform the desired task.

The manipulator command signals generated in this local loop are transmitted over the narrow band communication link to the remote real manipulator control loop. There the commands are realized in motion at high precision. The actual manipulator motion observed via a camera and/or by the manipulator sensors is transmitted back to the local loop over the narrow band communication link and used by the image operator to update the local loop model (Tan 1993).

One major difference between this model based manipulator control system and that of the free swimming vehicle is that the precision of the remote manipulator control loop is very high compared to that of the vehicle control.

Manipulators to be used on free swimming vehicles have different design specifications than manipulators for use in an industrial environment. In addition to being designed for a high pressure salt water environment (pressure 200 bar) the arm has to combine light weight with high strength and precision. In the MOBATEL project a study has been undertaken as a basis for the design of an underwater manipulator (Elle, Johnsen and Lien 1994).

\section{The MOBATEL laboratory}

In order to test the different methods developed for model based control and image processing as described above a laboratory environment has been developed called the 


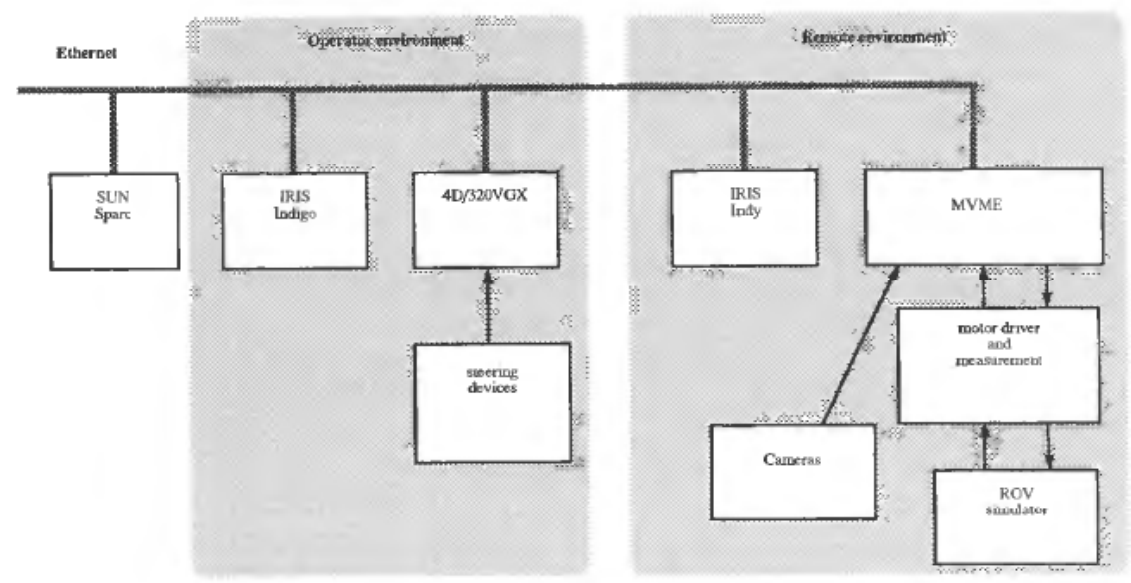

Figure 6.

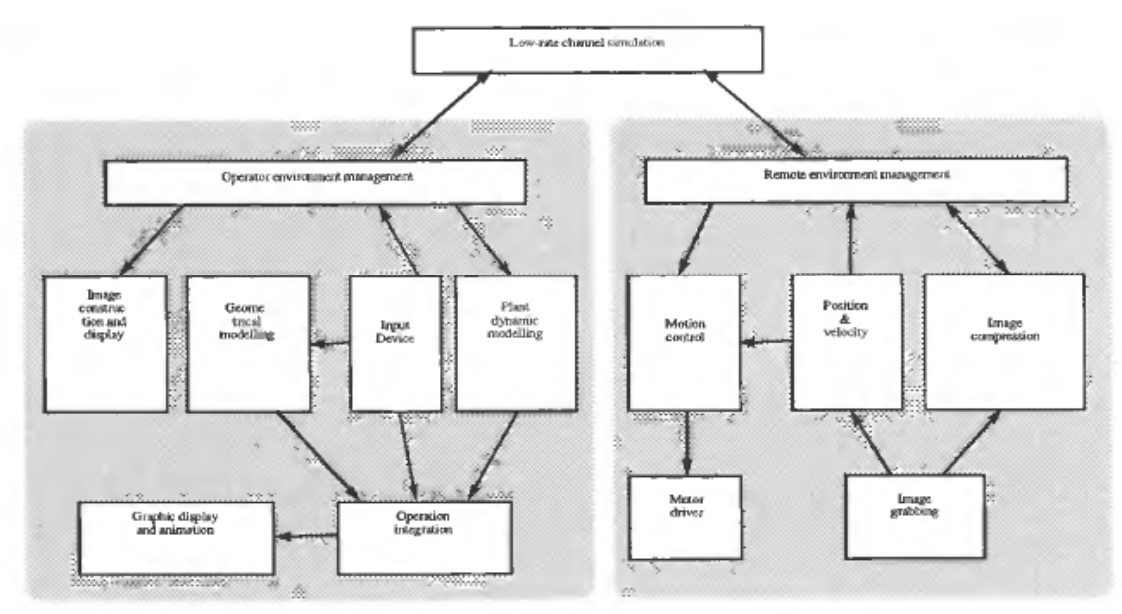

Figure 7.

MOBATEL-lab. It consists of a 6 DOF mechanical motion simulator with 6 independent servo drives capable of simulating motion in a volume $6 \times 10 \times 1.5 \mathrm{~m}^{3}$. By using a scale $1 / 10$, this represents a volume of $60 \times 100 \times 15 \mathrm{~m}^{3}$. In this volume are located objects of realistic size and shape which can be observed from a camera mounted on the motion simulator.

The motion simulator is controlled from an operator cockpit with the appropriate displays and joysticks for the image operator and the pilot as shown in Fig. 2. The computer control and image processing hardware setup is described in fig. 6 together with the software architecture shown in fig. 7.

\section{Conclusions}

The MOBATEL project will terminate in 1996. At that time 11 doctoral candidates will have finished their doctor of engineering degrees (doktor ingeniør) with research projects as described above. Their results are believed to be of major importance for future practical realization of model based control of subsea operations at great depths where the use of tethered vehicles may be difficult. 


\section{ACKNOWLEDGEMENTS}

The MOBATEL project is supported by The Research Council of Norway on contract 101022/420 and by Statoil, Saga Petroleum, Simrad Subsea, Bentech Subsea, Seatex and Silicon Graphics.

\section{REFERENCES}

Balchen, J. G. and Yin Shi (1994). The MOBATEL Project. Rep. 1994:129 W, Dept. of Engineering Cybernetics, The Norwegian Inst. of Technology, Trondheim, Norway, 1994.

Elle, O. J., JohnSEN, K. and LIEN, T. K. (1994). Mechanical analysis of high precision manipulators. Scandinavian Symposium on Robotics, (R. Tuokki, Ed.), Tampere University of Technology, Finland, October 4-6, 1994.

FJELlstAD, O.-E. (1994). Control of Unmanned Underwater Vehicles in Six Degrees of Freedom: A Quaternion Feedback Approach. Dr.ing. thesis, Rep. 1994:92-W, Department of Engineering Cybernetics, The Norwegian Institute of Technology, Trondheim, Norway.

Fossen, T. I. (1991). Nonlinear Modelling and Control of Underwater Vehicles. Dr.ing thesis, Rep. 1991:42-W. Department of Engineering Cybernetics, The Norwegian Institute of Technology, Trondheim, Norway.

Melen, T. (1994). Geometrical Modelling and Calibration of Video Camera for Underwater Navigation. Dr.ing. Thesis, Rep. 1994:103-W, Department of Engineering Cybernetics, the Norwegian Institute of Technology, Trondheim, Norway.

Spangelo, I. (1994). Trajectory Optimization for Vehicles Using Control Vector Parametrization and Nonlinear Programming. Dr.ing. Thesis, Rep. 1994:111-W, Department of Engineering Cybernetics, The Norwegian Institute of Technology, Trondheim, Norway.

SCHJøLBERG, I. and FOSSEN, T. I. ( 1994). Modelling and Control of Underwater Vehicle/Manipulator. Proc: Conference on Manoeuvring and Control of Marine Craft, (G.N. Roberts and M.M.A. Pourzanjani, Eds.), Maritime Research Centre, Southampton Institute, UK, 1994.

TAN, QING (1993). Modelbased Anthropomorphic Robot Telemanipulation. Dr.ing. Thesis, Rep. 1993:144-W, Department of Engineering Cybernetics, the Norwegian Institute of Technology, Trondheim, Norway.

REn, GuANG (1993). Finite Element Modelling the Hydrodynamic Environment of Autonomous Underwater Vehicles. Dr.ing. Thesis, Rep. 1993:9-W, Department of Engineering Cybernetics, The Norwegian Institute of Technology, Trondheim, Norway.

VOLDEN, RUNE (1995). Modelbased and Operator assisted Computer Vision in ROV Teleoperation. Dr.ing. Thesis, Rep. 1995: 1-W, Department of Engineering Cybernetics, The Norwegian Institute of Technology, Trondheim, Norway.

YIN, SHI (1993). An Investigation of Image Capture, Compression and Feature Extraction Algorithms, for an Underwater Telerobotics System. Dr.ing. Thesis.

Report 157-W, Department of Engineering Cybernetics, The Norwegian Institute of Technology, Trondheim, Norway. 